\title{
Collaborative Design Practice Involving Partners for the Advancement of Collaborative Engineering Education (PACE)
}

\author{
J. Mikkelsen ${ }^{1}$, A. Steeves ${ }^{1}$, W.L. Cleghorn ${ }^{2}$, P. Bastani ${ }^{1}$, R. Pattani ${ }^{2}$, and T. Pickles ${ }^{2}$ \\ ${ }^{1}$ Department of Mechanical Engineering \\ University of British Columbia \\ mikk@mech.ubc.ca \\ ${ }^{2}$ Department of Mechanical Engineering and Industrial Engineering \\ University of Toronto \\ cleghrn@mie.utoronto.ca
}

\begin{abstract}
This paper describes efforts to develop a collaborative design project involving third year mechanical engineering students from the University of British Columbia (UBC) and the University of Toronto ( $U$ of T). Selected students enrolled in a core kinematics and dynamics course at $U$ of $T$ were partnered with selected students enrolled in a core machine design course at UBC. These project groups were given the task of designing an automotive product specified by the industrial client, General Motors. The pilot project required students make full use of the advanced design resources provided under the Partners for the Advancement of Collaborative Engineering Education (PACE) program. This pilot project was performed as a simulation of real world automotive design where design offices around the globe participate in concurrent design of new automobile components and systems.
\end{abstract}

\section{Introduction}

It is not surprising that computers have become the universal tool for engineers. The computer has revolutionized the design process. A computer can now routinely perform design tasks that only a few decades ago were regarded as "wishful thinking." Yet these rapid changes in computer hardware and software has made it difficult for engineering schools to maintain current hardware and software tools for student usage. As a means to address these challenges, the Partners for Advancement of Collaborative Engineering Education (PACE) was established by General Motors and several of their computer vendors with selected Universities around the world to supply valuable design and analysis tools at minimal cost. At present, there are over
50 partner institutions around the world of which seven are located in Canada (University of British Columbia, University of Toronto, University of Waterloo, University of Ontario Institute of Technology, Queen's University, McMaster University, and Dalhousie University).

According to the official PACE website, the "Partners for the Advancement of Collaborative Engineering Education (PACE) links GM, Autodesk, EDS-an HP company, HP, Siemens PLM Software, Sun Microsystems, and their global operations, to support strategically selected academic institutions worldwide to develop the automotive product lifecycle management (PLM) team of the future. PLM, as it relates to PACE, is an integrated, parametricsbased approach to all aspects of a product's lifefrom its design inception, through its manufacture, marketing, distribution and maintenance, and finally into recycling and disposal. Currently, PACE is focused on: Requirements and planning (concept development), Styling (conceptualization), Product engineering (detailed engineering design), Simulation (validation, optimization), Manufacturing engineering (tooling, machining, 3D plant layout), and Managed development environment (product data management, supply chain collaboration, digital collaboration)" ${ }^{1}$

Current products donated to these institutions include powerful dual processor HP computer work stations complete with high speed graphics cards and a CAD specific operating mouse. In addition to the hardware, full operating licenses for software include UGS NX and MSC Adams for CAD modeling and analysis, ANSYS and FLUENT for detailed fluid flow and stress analysis, and Teamcenter Community for communication, file sharing and file storage. 


\section{Review of Classical Design Process}

The concept of engineering design is relatively new. The literature suggests that although formal engineering, as defined as the practical application of pure science and math concepts, can be traced back several hundreds of years, formal design approaches were introduced around the time of the Industrial Revolution. One of the earliest approaches to design was in the design of heavy industrial equipment such as railway locomotives and ships. The common element of a design process is to develop a product whose final functions meet the client's expectations and requirements.

The simplest form of the design process is the sequential design process (sometimes referred to as point to point design). This simple design process (Figure 1) can be summarized as a "sequential application of analyses based on the Newtonian principles of reductionism, mechanism and synthesis utilizing the intuitive skills of the designer." "2 Generally speaking, once a decision is made in this design process all subsequent design steps are constrained by this decision.

\section{Design Process}

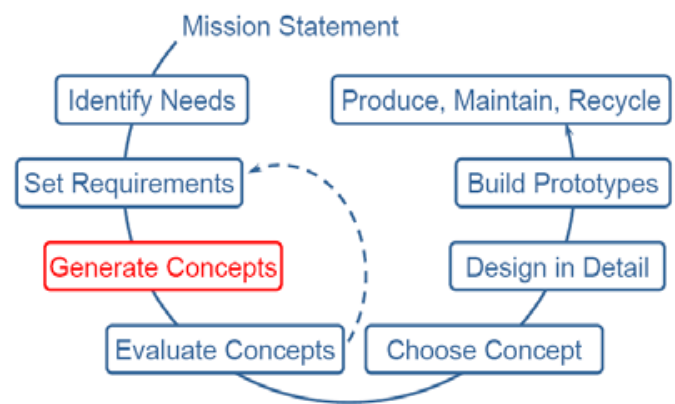

Figure 1. Traditional Sequential Design Process.

Further concepts in the scientific design processes have been proposed, developed and modified, with the design spiral concept being one of the most extensively used processes up until the development of the fast computer computational system and optimization techniques. In 1959, Evans ${ }^{3}$ first visualized and modeled the process for ship design, and is now known as the "Ship Design Spiral" (Figure 2).

The spiral representation depicts the iterative nature of the design process (not just ship design). The process begins with the owner requirements and proceeds through the nodes of the spiral towards the detail design. Since each node can be complex and interdependent, it is often laborious and expensive. There have been several refinements made to the design spiral over time, but the basic features have remained unchanged.

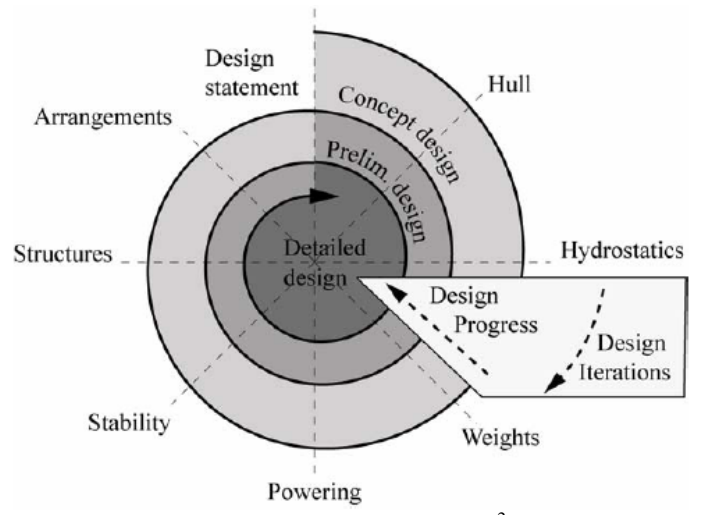

Figure 2. Ship design spiral ${ }^{2}$

Mistree et a1. ${ }^{2}$ states that there are two salient features of the design spiral. Firstly, while spirals represent converging towards a product, the process is divergent with respect to information and the increasing detail of definition. Secondly, it is recognized that when the spiral was first formalized, it represented a descriptive model that portrayed how design was done. It represented both the state-of-art and the state-of-industry at the time.

Limitations of the design spiral are that the process of design relies on sequential activity and iteration. This traditional approach is slow and no longer competitive in the open market. Using this concept directly with the computer means that the computer is used as a high speed calculator rather than as an integral design tool. Though the spiral approach may result in a satisfactory design it does not promote the identification of superior solutions.

\section{Concurrent Engineering Process}

Concurrent engineering requires that all elements of a product are integrated into a feedback design process (Figure 3). In this process changes in one design element may impact several other elements simultaneously. Other terms for this process are simultaneous engineering, Unified Life Cycle Engineering (ULCE), and producibility engineering. A formal definition for concurrent engineering is given by Winner" as "Concurrent engineering is a systematic approach to the integrated, concurrent design of products and their related processes, including manufacturing and support. This approach is intended to cause the 
developers, from the outset, to consider all elements of the product life cycle from conception through disposal, including quality, cost, schedule, and user requirements." ${ }^{4}$

In concurrent engineering, the initial design stages are especially significant because major decisions are often made based on a predominance of soft information (based on the designer's judgment and experience rather than proven principles and evidence). This can have far-reaching effects on the system being designed. Unlike the sequential design process, the information flow in concurrent engineering is bidirectional and decisions are based on both upstream and downstream considerations.

In concurrent engineering design, as decisions are made, the freedom to make further changes is reduced and the knowledge about the object of design increases. There is a progression from "soft" to "hard" information. As shown on Figure 4, this movement to hard information "drags" the knowledge curve to the left, improving the "quality" of information at early stages and allows for designs that are completed in less time and at less cost than those designed using a traditional sequential process ${ }^{2}$.

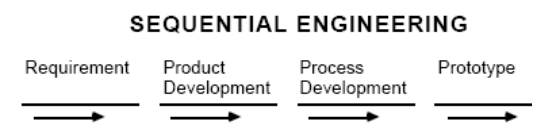

\section{CONCURRENT ENGINEERING}

Requirement

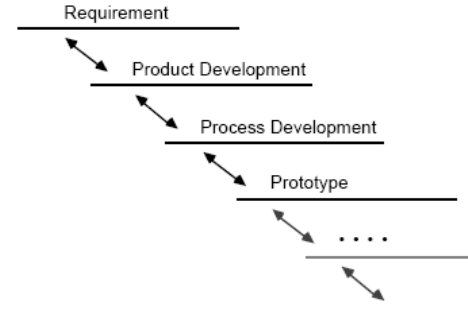

Figure 3. Sequential Versus Concurrent Engineering Design. ${ }^{2}$

According to Winner ${ }^{4}$, some of the reported benefits of concurrent engineering design are:

- The quality of designs was improved resulting in dramatic reductions of engineering change orders (greater than 50\%) in early production. - Product development-cycle times were reduced by $40-60 \%$ over the sequential design process.

- Manufacturing costs were reduced by $30-40 \%$ when multifunction teams integrated product and process designs.

Winner ${ }^{4}$ reports that the generic elements of concurrent engineering include:
- Reliance on multifunction teams to integrate the design, manufacturing and support processes of a product.

- Use of computer-aided design, engineering and manufacturing methods to support design integration through shared product and process models and data bases.

- Use of a variety of analytical methods to optimize a product's design, manufacturing and support processes.

- The costs of scrap and rework were reduced by $75 \%$ through product and process design optimization.

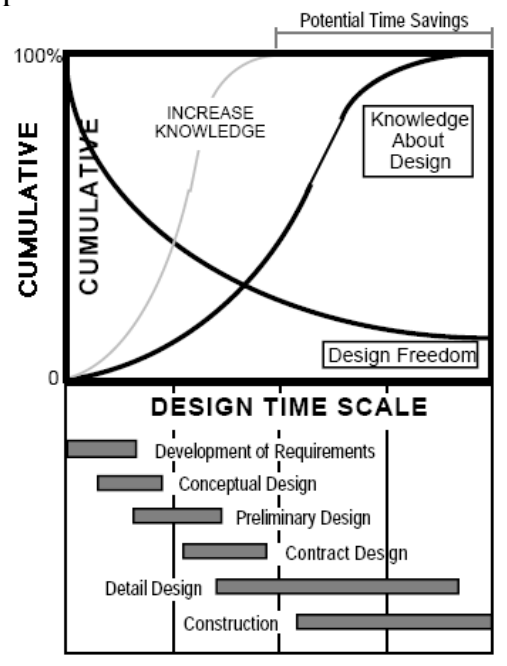

Figure 4. Design Knowledge Gained During Concurrent Design Process ${ }^{2}$.

"Compared to traditional engineering design in which synthesis of the product plays the central role, the synthesis of the process (which includes design, manufacture and support aspects) is the dominant feature in concurrent engineering. With the synthesis of the process at this higher level, the synthesis of the product follows naturally." It is obvious that computer resources are integral in a concurrent engineering process and can be considered a challenge to integrate within a design course.

\section{Overview of Vehicle Design}

A traditional car design process is shown on Figure 5. The traditional sequential design usually begins with a statement of intent to design and manufacture a specific vehicle. A set of design specifications is developed from customer market surveys and competing products analysis. Concepts are developed and eventually the detailed design phase leads to 
prototypes to test system integration, manufacturing, and eventual certification before a formal market launch.

One may noticed that the preliminary design stage is missing. However, the tasks previously accommodated in the preliminary design stage are often distributed across conceptual and detailed design. Prototypes are tested during the detailed design stage and the results are fed back into the design for system integration evaluation and optimization.

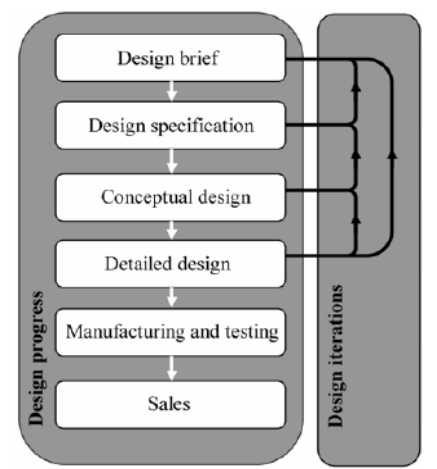

Figure 5. Traditional Vehicle Design Process 5 .

A further development in vehicle design is the set based design which has been credited to Toyota. Set based design comprises of the standard engineering design stages such as the concept, preliminary and detailed design. However, it delays the selection of the favorite design concept to the testing of prototypes. By doing so thereby allows to gradually narrowing the solution set towards a more global optimum. A great number of design teams work concurrently on detailed design solutions. The weakest solutions will be eliminated at the end of each design stage and the most favorable sets recombined, modified and detailed. The method helps to avoid costly design iterations. The method also allows tolerances and fixed points to be delayed significantly. Set based design has been adopted by several automobile manufacturers.

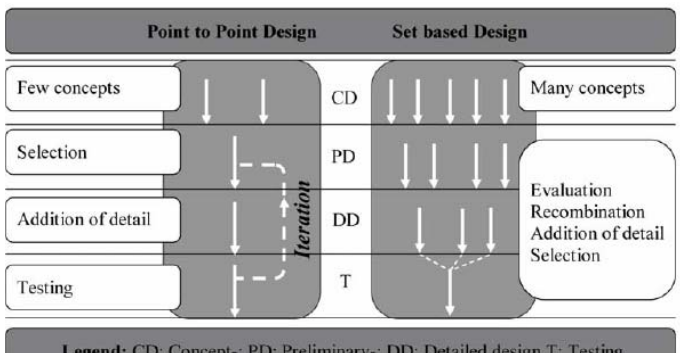

Figure 6. Set Based Design Process 5 .

\section{Example of PACE Sponsored Student Project}

Undergraduate mechanical engineering students from the University of British Columbia (UBC) and University of Toronto (U of T) were given the opportunity to work together to complete a collaborative design project for the first time in the fall of 2007. Teams were tasked to design a simplified front suspension system given that the general suspension dimensions, simplifying assumptions, and values for the deflection of the suspension strut in response to an applied force were provided by the General Motors engineers. Teams were given design criteria where the suspension must survive the impact with a certain size and shape pothole. The objective of the project was to optimize the design of the suspension system to minimize the vertical force in the strut during a pothole event at $40 \mathrm{~km} / \mathrm{h}$. Additional objectives included optimization for manufacturability, design for durability, and an assessment of human factors to ensure the comfort of passengers.

In addition to the industrial context in which this problem was created, this project was made particularly remarkable because of the composition of the design teams. Twelve students from UBC and six students from $\mathrm{U}$ of $\mathrm{T}$ formed three design teams; each team was made up of four UBC and two $\mathrm{U}$ of T students. The hybrid teams allowed for students to harness the diverse educational backgrounds and experience made available by different institutions. UBC students provided a great deal of experience in static analysis, solid modeling, and design for manufacturability, while the $\mathrm{U}$ of $\mathrm{T}$ students provided an understanding of kinematic and dynamic analysis. A review of the top team for 2007 is presented as example work.

As stated earlier, one of the goals of the PACE program is to expose students to modern computer design tools. In this project several technical software packages were employed to complete this project. These included UGS NX and MSC Adams for CAD modeling and analysis; MATLAB and CES Material Selection used to confirm results and to meet design objectives.

UGS NX was utilized primarily in two ways. The UBC students used NX Modeling to create a solid model of the suspension system for which analysis could be conducted (Figure 7) while the $\mathrm{U}$ of $\mathrm{T}$ students made use of $\mathrm{NX}$ Motion to conduct analysis to determine the strut 
forces during impact with the pothole. From initial results, the students developed a more comprehensive analysis using MSC Adams. With some external help, the $\mathrm{U}$ of $\mathrm{T}$ students were able to develop and use the detailed Adams model to optimize the damping coefficient, and initial strut angle of the suspension system (Figure 8). However, the students did question the results due to the many assumptions and uncertainties in the model.

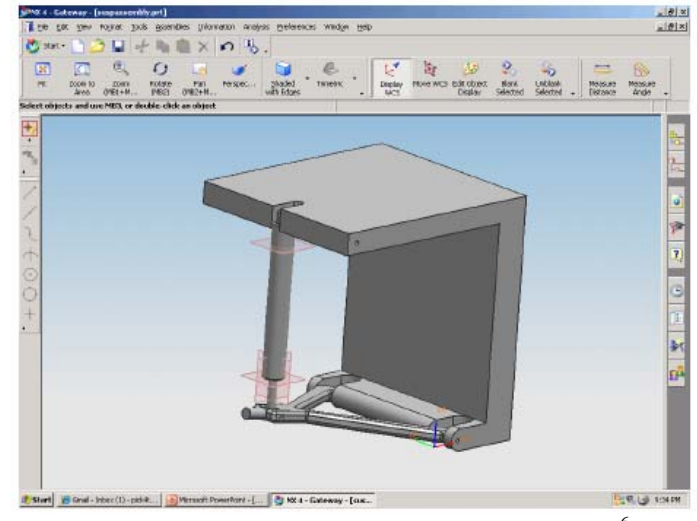

Figure 7. UGS NX Model of Suspension System ${ }^{6}$

The UBC students also used additional software packages: the well known MATLAB program and CES Material Selection. MATLAB was leveraged to create complex mathematical models to optimize key parameters while CES Material Selection was used to determine the most appropriate materials to manufacture each component while balancing required performance and cost.

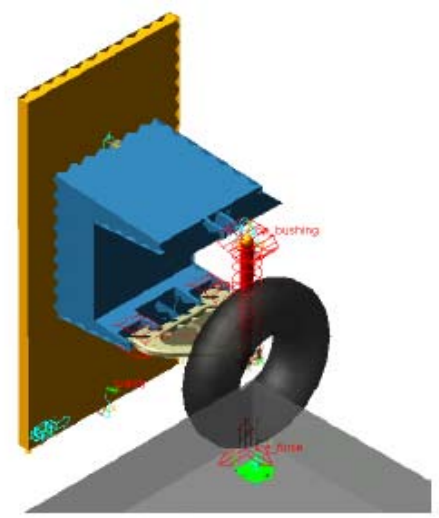

Figure 8. MSC Adams Analysis Model ${ }^{6}$

Given that the project teams are composed of student members separated by thousands of kilometers, communication became a large challenge. It was often difficult to coordinate meetings due to conflicting schedules and the three hour time difference between members of the same team. Therefore, it was critical to make effective use of the communication tools available through the PACE Program. UGS Teamcenter Community, based on the familiar Microsoft Share Point platform, offered design teams a resource that allowed for Document Management and communication in a central environment. The homepage (Figure 9) provided users with access to pertinent information. Teamcenter Community replaced email as a communication tool in the project. However, the students reported that some tasks still required the use of more traditional communication methods.

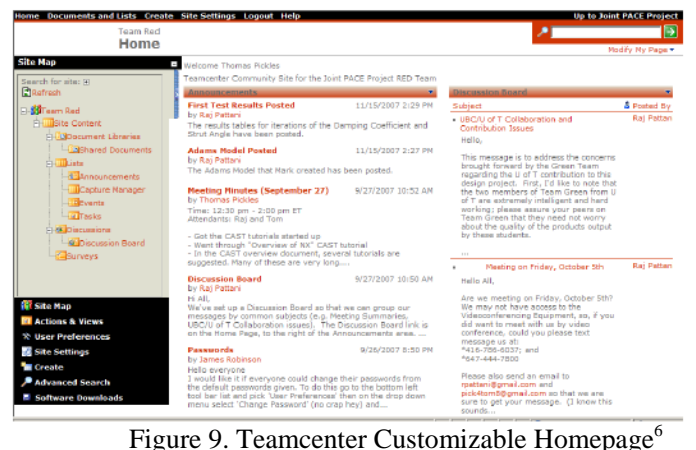

The Document Management system, (Figure 10) was perhaps the most useful feature of Teamcenter Community as it allowed for design teams to use the same version of a document when making revisions. For example, students were able to post revisions to one central location, rather than having several versions floating in team members' email accounts.

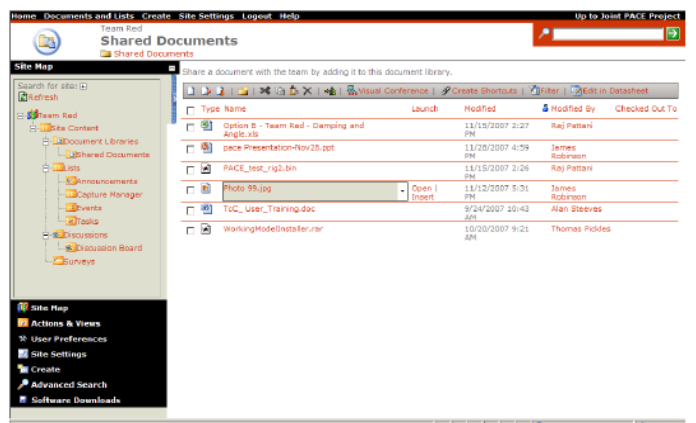

Figure 10. Teamcenter Document Management System ${ }^{6}$

The students reported that an important feature of Teamcentre Community was the Discussion Board. This feature allowed for the teams from the two academic institutions to more easily provide status reports. This was 
particularly important because the $U$ of $T$ teammates needed to update their Team Leaders on the UBC side frequently, and the UBC students needed to update the $\mathrm{U}$ of $\mathrm{T}$ students with new design decisions that may have been made between regular meetings. The Discussion Board also allowed for students to organize their discussion into various areas. For example, message threads were created for "Status Reports," "Meeting Availability," and "Suggestions for the Final Presentation." However, the students' familiarity with email meant that students used email more frequently in their day-to-day work, and so, for more timesensitive matters, email was used as the primary mode of communication. In industry, Teamcenter Community is likely more pervasive in a corporation's operations, and so it is expected that this would not be an issue.

Videoconferencing was used sparingly in the earlier stages of the project but extensively to prepare and deliver the final presentation. The videoconferencing technology allowed each team to present their final analysis and design as one cohesive unit to the judges, who were all located in the $U$ of $T$ presentation room. The UBC students were displayed on a large presentation screen and could be seen and heard clearly by the judges. The PowerPoint presentation was shown on an adjacent screen, giving the impression that the presenters were standing next to each other. As well, the judges were visible on the UBC screen, allowing the UBC students to gauge the judges' interest and reactions. Despite a few drawbacks with the size of the video conference room, the videoconferencing technology allowed for effective presentations to be delivered by teams composed of students on opposite sides of the country.

The students reported that although the ability of videoconferencing to provide personal and interactive team meetings, the technology was utilized only a few times during the earlier project stages. Although a kick-off meeting and a few other intermittent sessions were held, communication between team members was carried out most often using email, Teamcenter, and instant messaging. Although the many benefits of videoconferencing were understood, the technology was not used primarily for logistical reasons such as the time difference, the conflicting schedules of each of the team members, and lack of direct access to the equipment limited the use of this equipment.
A key component of the concurrent engineering practice is the application sharing tools within Teamcenter Community. Application sharing enables two or more users to access a shared application simultaneously from their respective computers in real-time. Control of the application can be toggled between computers allowing all remote users to interact, design, edit, and comment on a design or analysis in real time.

Despite the many potential benefits of application sharing, it was never utilized during the student project. The student team reported that since the application sharing was only available at the universities, common times had to be arranged during school hours to use this feature. In addition, none of the students felt very confident with the software. Thus, teams were more reluctant to attempt an application sharing session because of the steep learning curves required. This led resulted in assigning tasks for each school to be mostly independent of each other.

\section{Lessons Learned}

In this Collaborative PACE Design Project, mechanical engineering students from the UBC had a strong understanding of solid modeling and design for manufacturing, while $U$ of $\mathrm{T}$ students were able to provide kinematic analysis. Students from the two institutions were able to contribute to separate parts of the project because of the nature of the courses in which they were carrying out the PACE project. It was valuable for the students to collaborate so that these different areas of specialty could be brought together to provide a more thorough evaluation of the simplified suspension system and to ultimately provide an optimized design. Further, the project demonstrated a key benefit that collaboration of this kind provides: the ability to harness the knowledge and training of individuals from different regions to complete a single project.

First hand experience in the successful execution of such a project is particularly beneficial for engineering students. When these students enter professional engineering, it is important that they have an understanding of how to pool all resources, especially human capital, to complete work in as efficient a way as possible.

There are two areas of communication in which students were able to excel through this project: technical and core competence. With the 
development of new communication tools, students need to become experts in using a diverse range of communication modalities. This project provided students with the unique opportunity of learning how to use the Teamcenter Community platform. Videoconferencing technology used in this project allowed students to learn how businesses spread across many regions use these new tools to work together. The technical competence developed by the students in these two particular tools is not limited to the systems used in this project; the structure of the project itself forced students to adapt to a new team dynamic, and to creatively use the new tools that were available to them. Students became flexible with the methods in which they could communicate with each other: the use of newer technology allowed for greater collaboration than with traditional communication methods.

Further, and perhaps more importantly, students developed their core competencies in communication. Because of new limitations that were imposed as a result of constraints from time zones, conflicting work schedules, and the availability of videoconferencing equipment, students learned the importance of effective and efficient communication. Videoconferencing time needed to be used for situations where brainstorming or open discussion were required, while discussion boards or email needed to be used for more routine updates on the status of work at both institutions. During undergraduate education, it is particularly valuable for students to learn the importance of effective communication; the PACE project allowed students to learn this in a realistic environment.

The major benefit of introducing Collaborative Education at the undergraduate level of study lies in the realistic project experience it offers students. This project provided an engaging learning experience for students that allowed teammates to operate outside the confines of traditional coursework. Further, because the design problem was prepared by industry partners, it offered a practical way to apply learning in a realistic environment. The project offered context that perhaps would not have played an important role in an academic project; for example, students had to coordinate their work to ensure that components that were used in design and analysis were economically viable and safe for use in the automobile industry. This project successfully simulated professional work conditions more so than other academic projects.
As this was the first time either of the institutions had participated in a collaborative project like this there were a number of challenges to overcome for the next year. The students reported that the pre-project organization and communication of project requirements could be made clearer. Since two different academic courses were utilized for this pilot project, the expectations of the professors and administrators at $\mathrm{U}$ of $\mathrm{T}$ were different from those at UBC resulting in the workload, time commitment per week, course weighting, and common meeting times were not equal amoung the courses. This created some tension early on as the students' understanding of the project varied significantly between the two institutions. In the future, it is vital that the project has equal weightings for the students at both institutions. This will lead to a more balanced division of work and a better understanding of time commitment expectations. Common meeting times should be arranged beforehand to ensure good communication and management of video conferencing and computer equipment.

The project teams had the opportunity to work with exciting, powerful, and state-of-the art PACE-sponsored software packages. However, the teams had very little previous experience with packages such as NX and Adams; these programs are quite difficult to master in order to obtain meaningful results. Without proper training resources, much of the short three month project timeline was spent trying to learn the software. In future projects, the software learning curve must be taken into account. Training time should be incorporated into the project timelines; more importantly, though, formal training should be provided. A few hours of instruction from an expert user and the ability to keep in touch and ask software related questions would have significantly improved the teams' ability to use the packages. Software training should become a priority in future PACE collaborative projects.

In the end, teams were able to deliver successful final presentations. However, team cohesiveness was an issue throughout the majority of the project. It is very difficult to have an intertwined team over such large geographic distances, but it could be improved upon. First, videoconferencing should be used much more extensively. In conjunction with organizing the common meeting times each week, the videoconferencing time must be utilized even if no critical design issues need to be discussed. Videoconferencing allows for social interaction 
and bonding beyond just the technical details; this is vital to foster a successful, dynamic team.

The use of application sharing will give team members a greater understanding of concurrent engineering practice. In this project, application sharing was not utilized mainly due to the fact that it was not deemed necessary since each team's responsibilities were exclusive of each other. This leads into the third point that in the future the tasks at each institution should be more closely dependent on each other. In turn, this would encourage greater communication and more extensive use of communication tools such as Teamcenter and Application Sharing.

\section{Conclusions}

Increased globalization requires engineering teams to work together across large geographical distances. Creating a realistic global collaborative and concurrent design experience within an academic setting is especially challenging.

This project provided a unique experience for students in three ways: the opportunity to use state-of-the-art engineering design software such as NX Motion and MSC Adams; the ability to use communication tools such as Teamcenter Community and videoconferencing software; and, the opportunity to collaborate with students from an institution that was several provinces away on an industry sponsored project. The lessons learned and experience gained go far beyond what is offered in the classroom to offer a real-world-like team atmosphere that has already proven to be an extremely useful experience for the students as they strive towards becoming successful engineers. As the first attempt at such a collaborative project it was deemed a success. All the selected student members learned many valuable technical and communication skills.

Several improvements are proposed for future collaborative design exercises. These include improved course administration, improvements in communication tools, reducing the learning curve for students, and stressing the requirements for application sharing. Without developing this type of learning environment, it is difficult to mimic modern concurrent and collaborative design practice.

\section{Acknowledgements}

The University of Toronto and the University of British Columbia gratefully acknowledge the generous support from the Partners of the PACE Program for making this project possible. The successful delivery of this type of innovative project requires the dedication and effort of a large number of people. The authors wish to pay special thanks to the industrial partners, namely General Motors, for providing the design challenge and design reviewers. Finally, a special thanks to all the students enrolled in the program for their patience and hard work during the development of this new program.

\section{References}

1. Welcome to PACE Website, Partners for the Advancement of Collaborative Engineering Education, www.pacepartners.org.org/index July 2, 2009.

2. F. Mistree, W. Smith, B. Bras, J. Allen, and D. Muster, "Decision-based Design: A Contemporary Paradigm for Ship Design,” SNAME Transactions, Vol. 98, 1990.

3. J.H. Evans, “Basic Design Concepts”, Naval Engineers Journal, pp. 671-678, Nov. 1959.

4. Winner, R.I., Pennell, J.P., Bertrand, H.E., and Slusarczuk, M.G., "The Role of Concurrent Engineering Weapons Systems Acquisitions”, IDA Report R-338, Institute for Defense Analyses, Alexandra, Virginia, 1988.

5. Hopfe, C.J., Struck, C., Hensen, and J.L.M., Böhms, M., "Adapting Advanced Engineering Design Approaches to Building Design - Potential Benefits", Proceedings of the 6th Int. Postgraduate Research Conf. in the Built and Human Environment, Technische Universiteit Delft, $\mathrm{BuHu}$, University of Salford., pp. 369-378 April 2006.

6. Bastani, P., Pattani, R., and Pickles, T., "The 2007 University of Toronto/University of British Columbia PACE Collaborative Design Project” Proceedings of the $4^{\text {th }}$ Annual PACE Conference, PACE Partners, Detroit, Michigan, July, 2008 